\title{
MPF-LEACH: modified probability function for cluster head election in LEACH protocol
}

\begin{abstract}
In this research, we enhance the LEACH protocol by updating Cluster Head $(\mathrm{CH})$ election probability function (thresholds). More probability was given to an out-of-service $\mathrm{CH}$ to be elected again. The idea is to get benefit from $\mathrm{CH}$ residual energy in order to extend the network lifetime. A new threshold was introduced which guarantees a non-zero probability value of a $\mathrm{CH}$. We proposed a newly developed research technique to enhance the original LEACH protocol. The enhancement focuses on extending a WSN's lifetime, and increasing its throughput. It is achieved by giving more probability to re-elect the expired $\mathrm{CH}$ that has been removed from $\mathrm{CHs}$ list because of its insufficient residual energy. Several experiments were conducted to evaluate the efficiency of our proposed MPF-LEACH approach. From the experimental results, a remarkable enhancement in the network lifetime and throughput is achieved. We have improved the election probability threshold for the original LEACH protocol by benefiting from the $\mathrm{CH}$ residual energy. As a result, the whole network lifetime was increased due to the extra chance that is given to a $\mathrm{CH}$ to be elected again.
\end{abstract}

Keyword: Routing protocol; WSNs; Wireless sensors networks; Energy aware; LEACH protocol; Cluster head; Threshold election probability 